\title{
THE PROBLEM OF DOSING AND DELAYS IN THE PROCESS OF USAGE OF ECONOMIC POLICY INSTRUMENTS
}

\author{
REVAZ GVELESIANI \\ Doctor of Economic Sciences, Professor, \\ Ivane Javakhishvili Tbilisi State University, \\ Georgian-German Institute of Economic Policy, \\ Academician of the Georgian Academy of Economic Sciences, Georgia \\ revaz.gvelesiani@tsu.ge
}

\section{GIVI BEDIANASHVILI}

Doctor of Economic Sciences, Professor

European University, Institute for Research of Economic and Social Problems of Globalization, Associate Professor, Ivane Javakhishvili Tbilisi State University, Academician of the Georgian Academy of Economic Sciences, Georgia g.bedianashvili@eu.edu.ge

Abstract. In order to make a rational decision in economic policy, it is necessary to examine and understand the importance of conformity tools towards the goal. This should be done in relation to the whole system of goals, including the goals that are recognized as core values. Criteria needed to reduce decision-making costs require a criterion that facilitates evaluation. We should also consider which tools are allowed to solve a specific problem based on the economic policy program. Such a program is a concept of economic policy. It provides us with sufficient and unambiguous information on substantive and politically significant consensus. It is a matter of consensus that the economy, as an integral part of a more inclusive system of public goals, has a subordinate place. This condition determines its value. Considering these circumstances, it is easy to determine whether the goal is conforming to the system. Conformity to the system is the criterion by which it is measured, which is the main thing in the concept of a given economic policy.

In determining the long-term socio-economic development strategy of the country in the process of economic policy-making, it is important to ensure the targeted orientation of macroeconomic parameters at the macro-systemic level. In addition, the level of the country's economy and social sphere, the state of entrepreneurial activity and the business environment in general, the peculiarities of cultural characteristics, the existing mechanism of socio-economic relations (institutions) and the peculiarities of value orientations of society should be taken into account.

KEYWORDS: GOALS AND INSTRUMENTS OF ECONOMIC POLICY, DECISIONS OF ECONOMIC POLICY, COMPLIANCE WITH GOALS, CONCEPT AND SYSTEM, THE PROBLEM OF KNOWLEDGE AND ASSESSMENT, DOSAGE LIMITS OF INSTRUMENTS.

For citation: Gvelesiani, R., Bedianashvili, G. (2021). The problem of dosing and delays in the process of usage of economic policy instruments. Globalization and Business, №11, 16-24. (In Georgian). https://doi.org/10.35945/gb.2021.11.002 


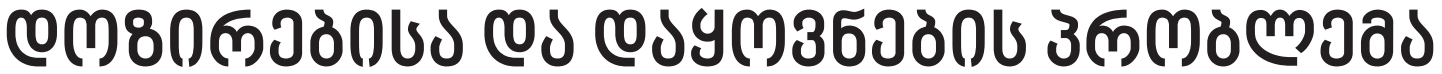

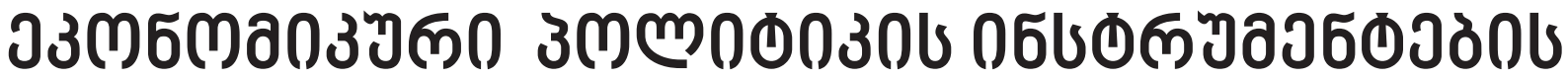

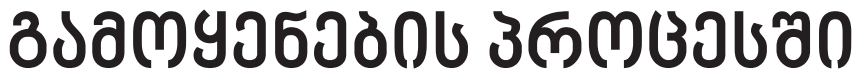

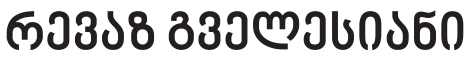

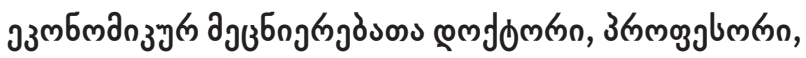

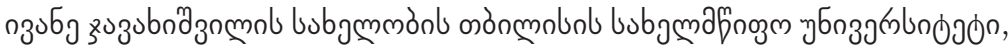

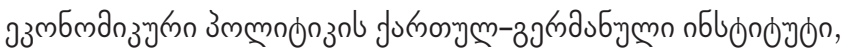

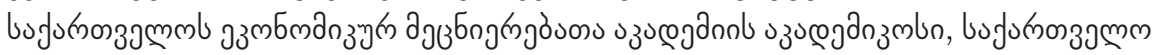

revaz.gvelesiani@tsu.ge

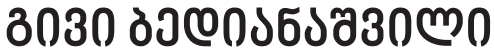

\section{गзмбмдаз}

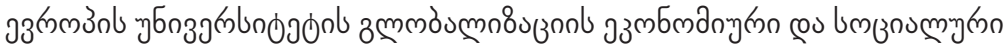

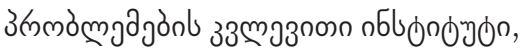

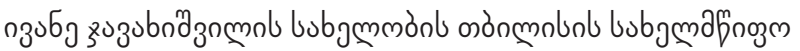

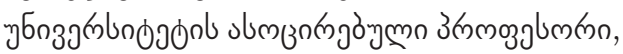

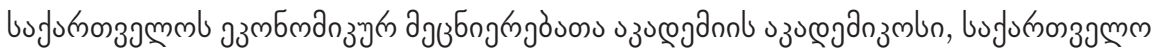

g.bedianashvili@eu.edu.ge

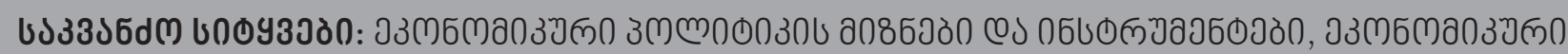

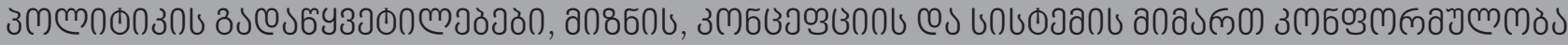

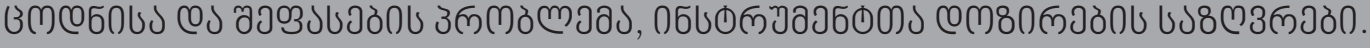

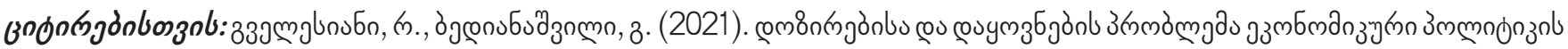

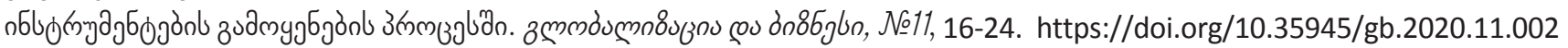

\section{ฆว৬১З১ल0}

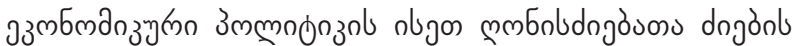

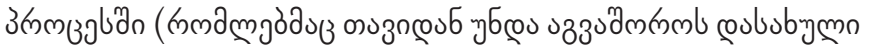

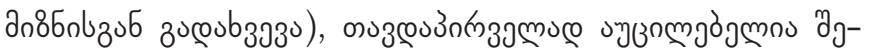

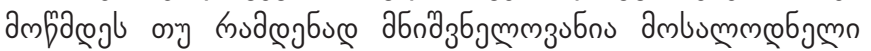

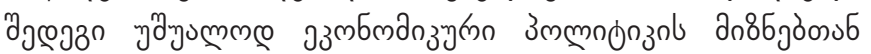

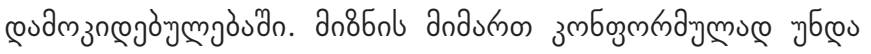

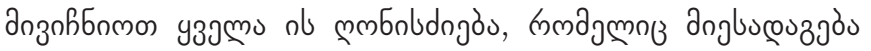

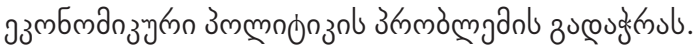

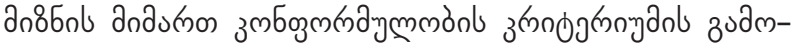

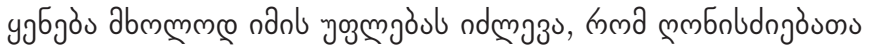

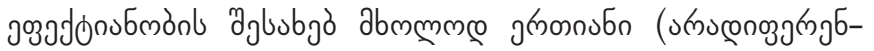

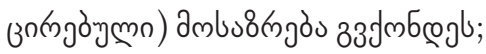

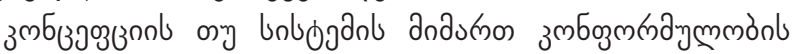

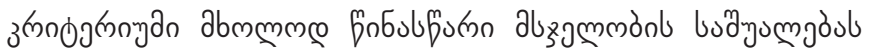

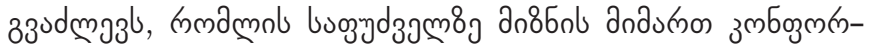

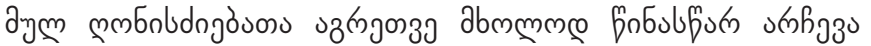
כonodmgos (Tuchtfeldt, 1982).

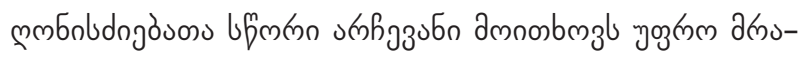

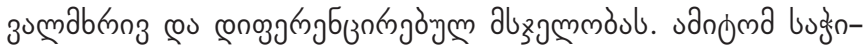

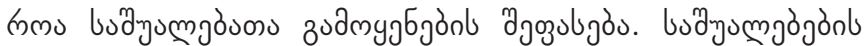

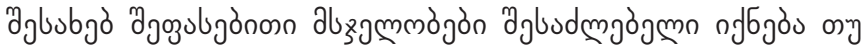

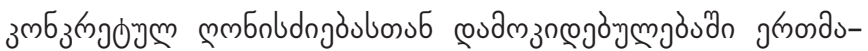

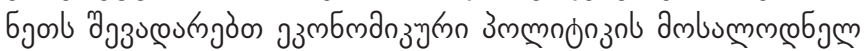

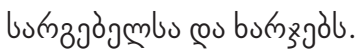

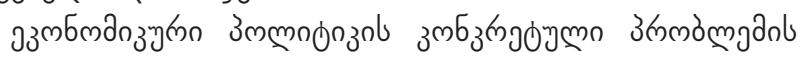

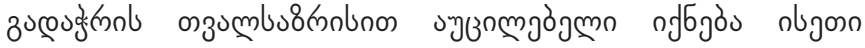

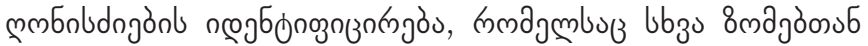

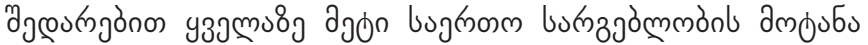
yjydmno.

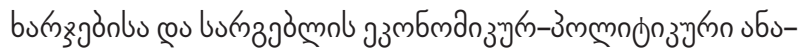

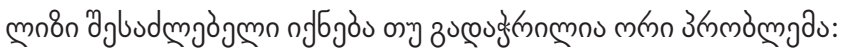

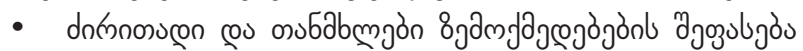

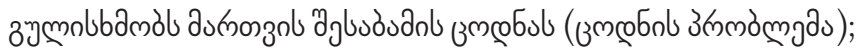

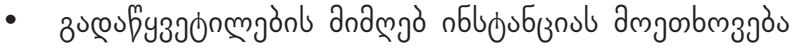

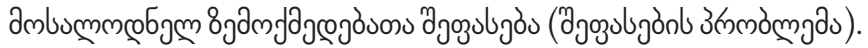




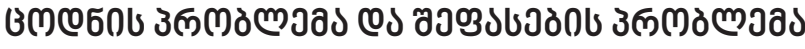

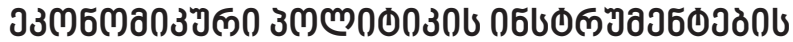

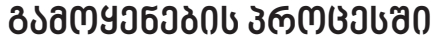

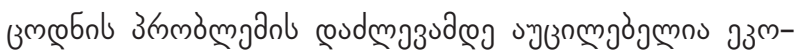

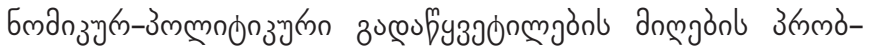

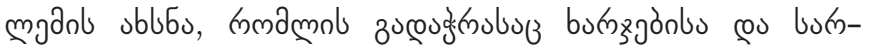

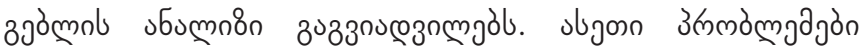

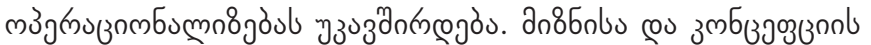

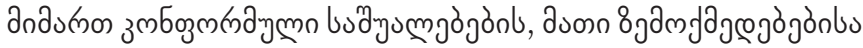

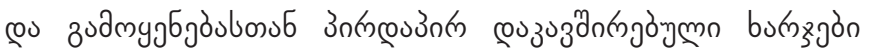

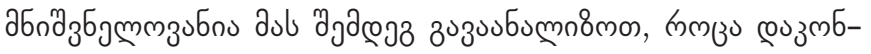

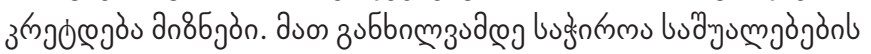

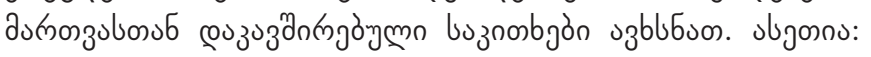

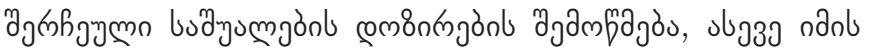

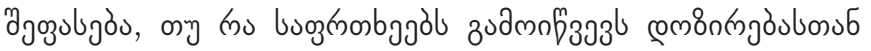

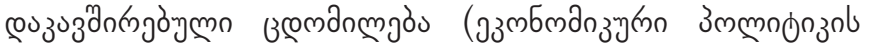

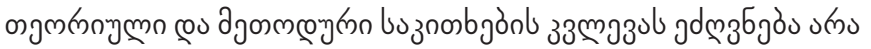

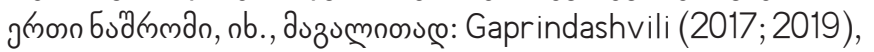
Gvelesiani\&Gogorishvili (2014), Gvelesiani (2015a;2015b; 2016a; 2016b; 2017a; 2017b; 2017c; 2020a; 2020b; 2020c; 2020d; 2021).

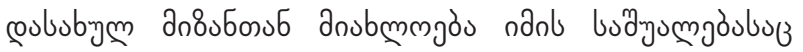

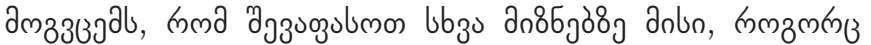

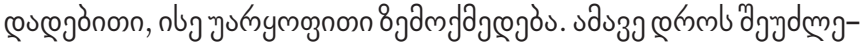

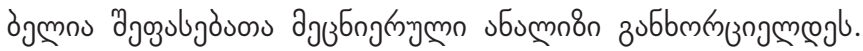

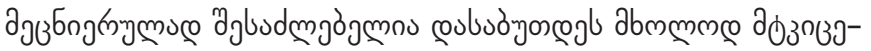

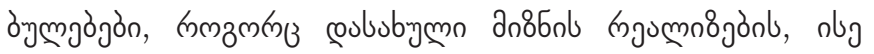

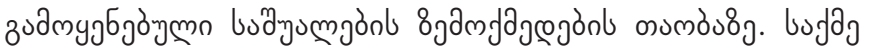
jbgos a

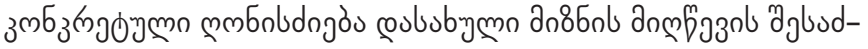

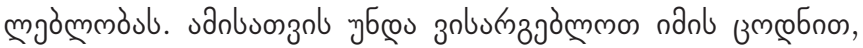

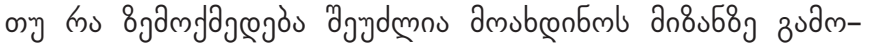

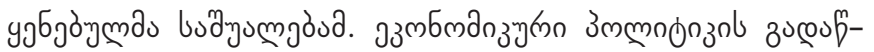

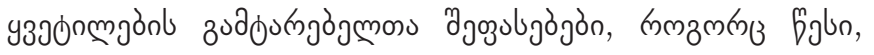

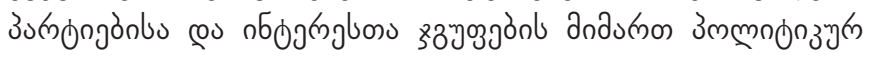

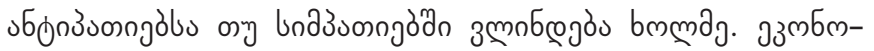

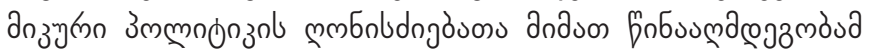

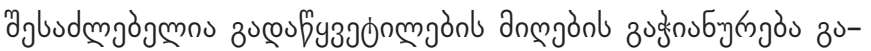

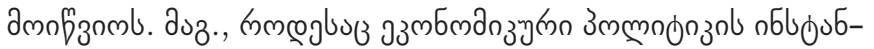

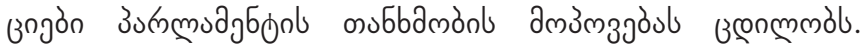

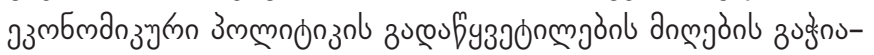

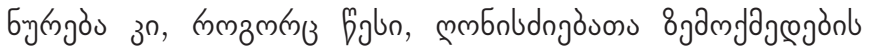

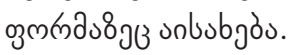

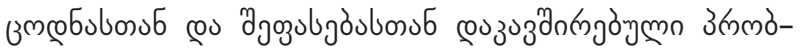

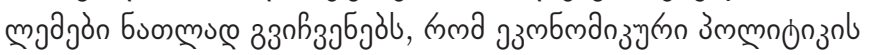

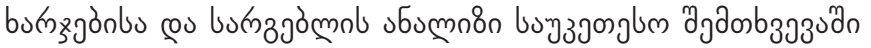
s nub

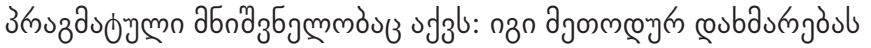

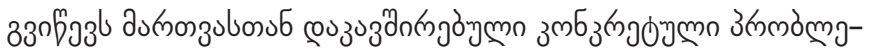

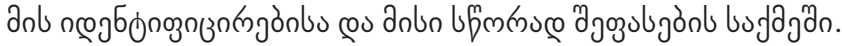

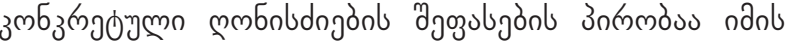

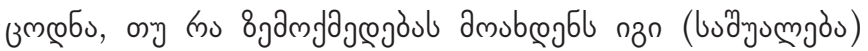

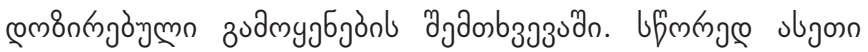

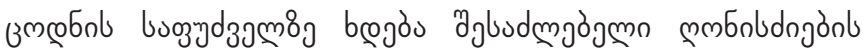

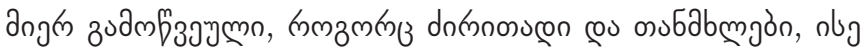

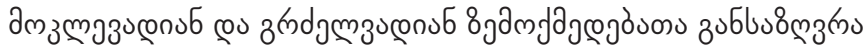

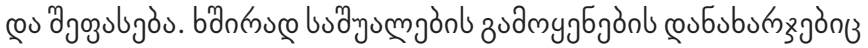

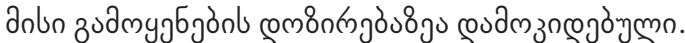

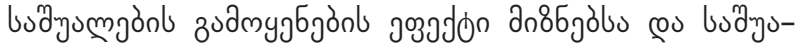

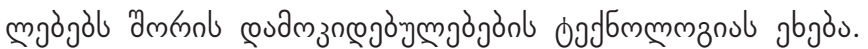

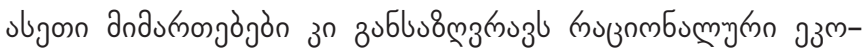

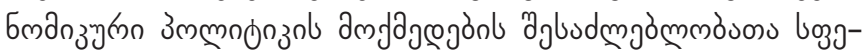

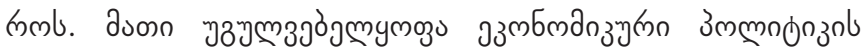

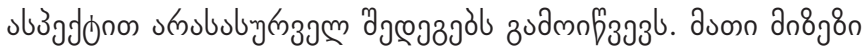

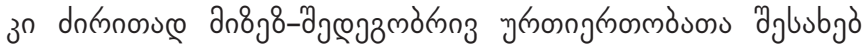

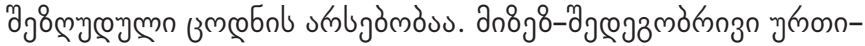

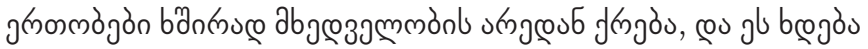
папома пма:

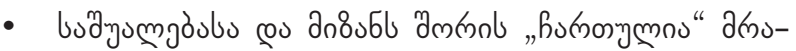

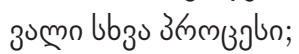

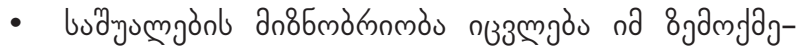

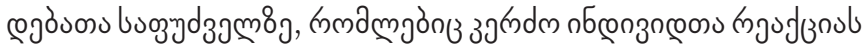

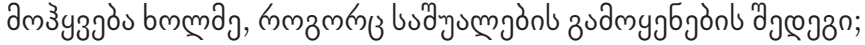

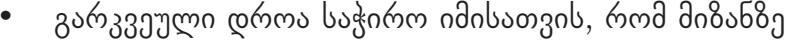

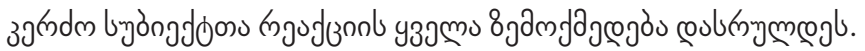

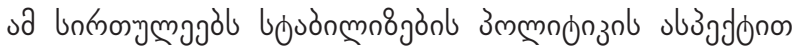

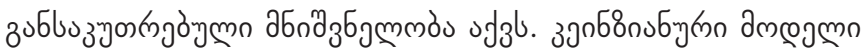

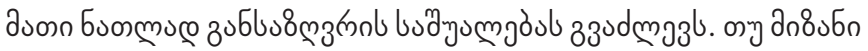

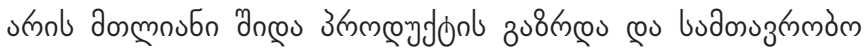

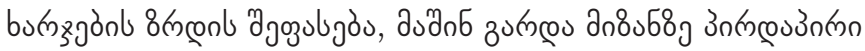

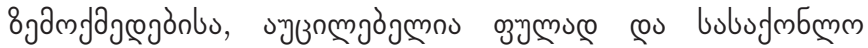

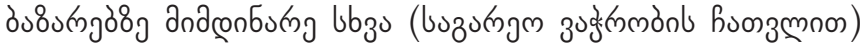

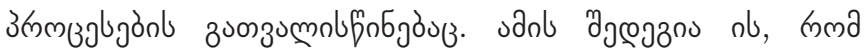

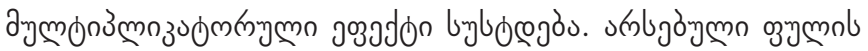

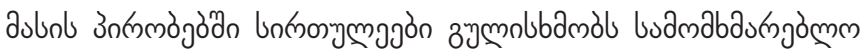

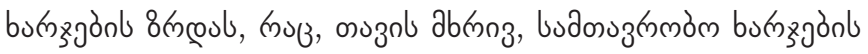

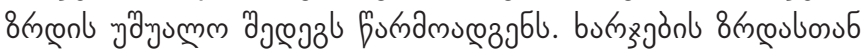

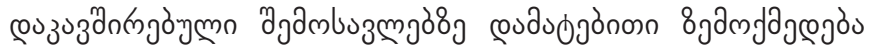

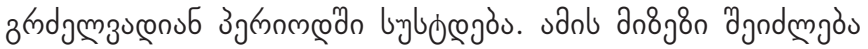

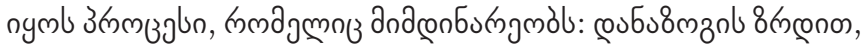

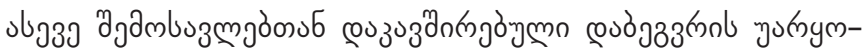

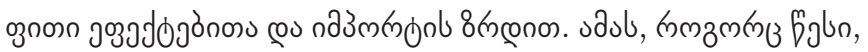

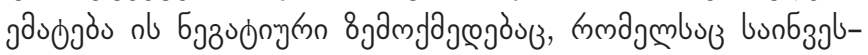

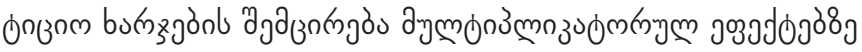

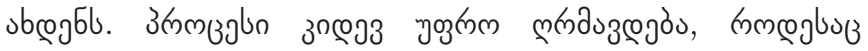

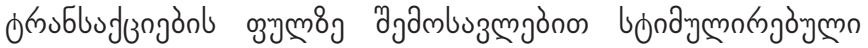

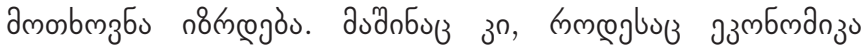

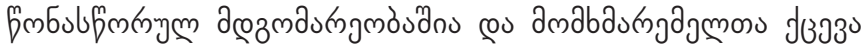

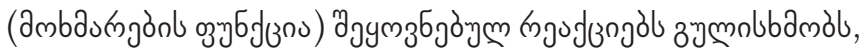




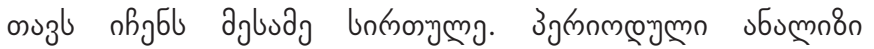

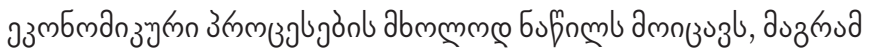

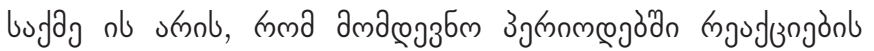

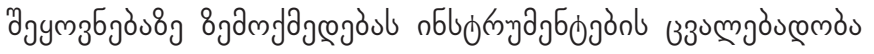

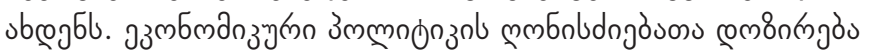

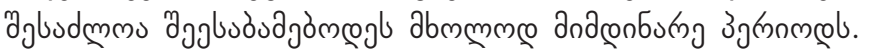

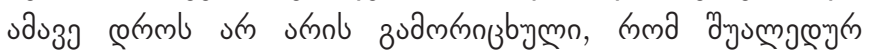

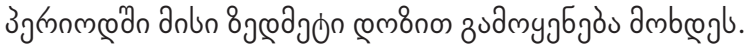

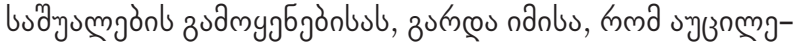

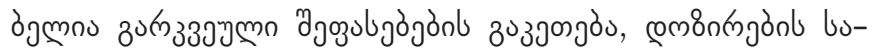

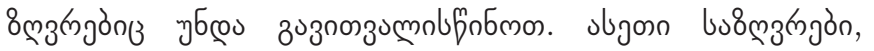

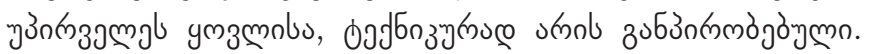

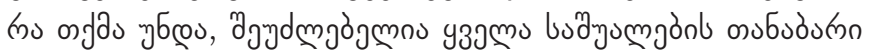

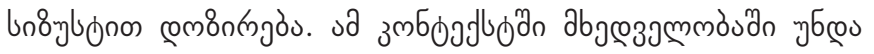

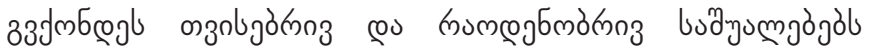

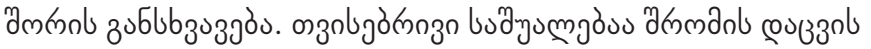

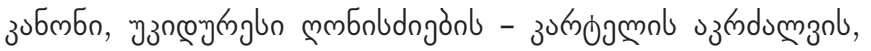

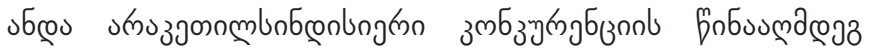

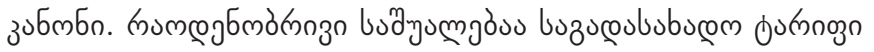

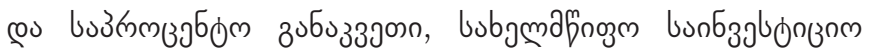

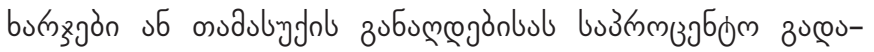

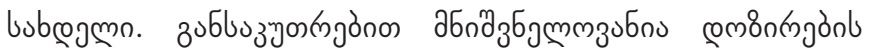

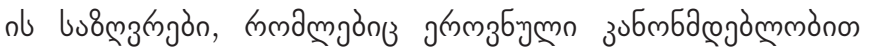

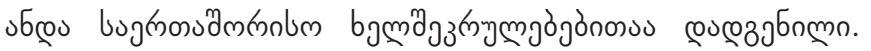

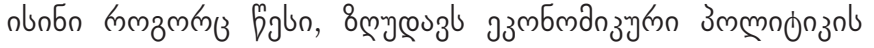

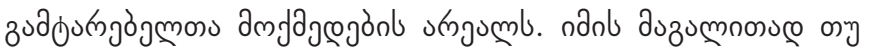

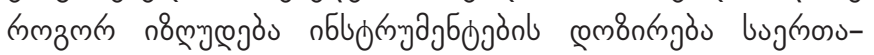

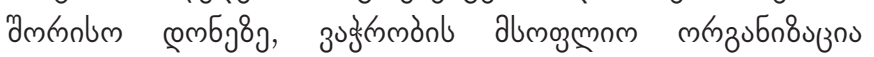

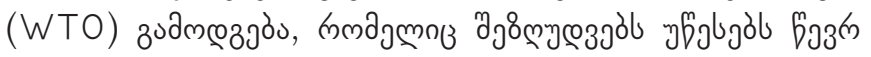

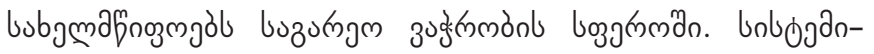

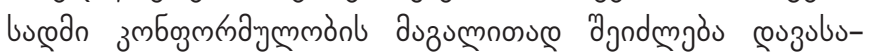

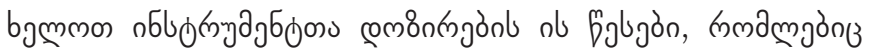

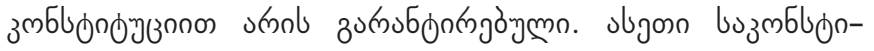

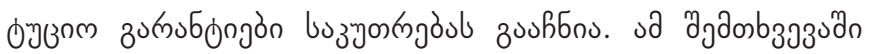

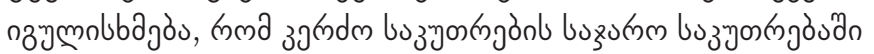

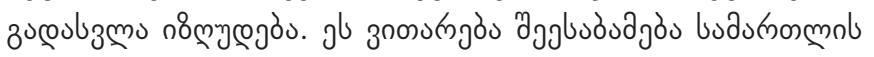

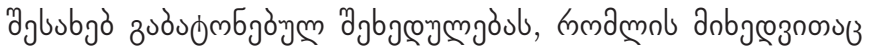

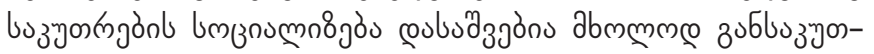

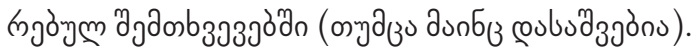

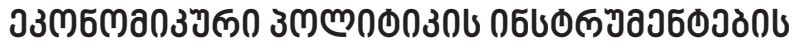

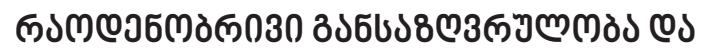

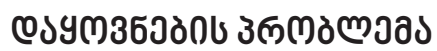

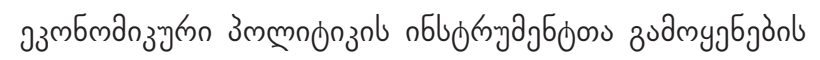

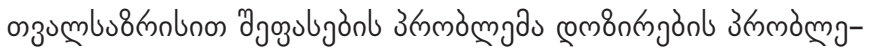

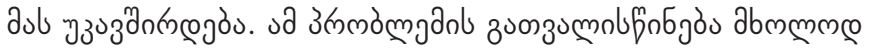

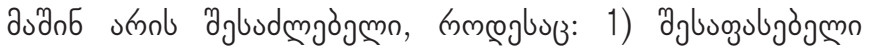

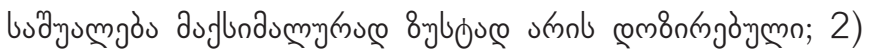

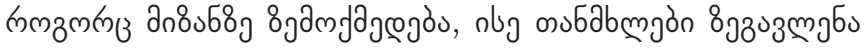

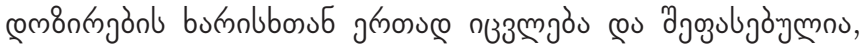

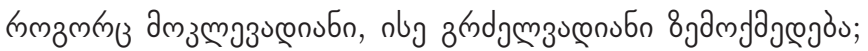

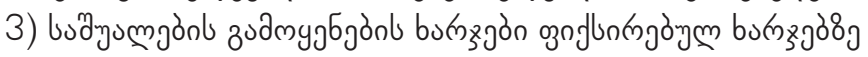

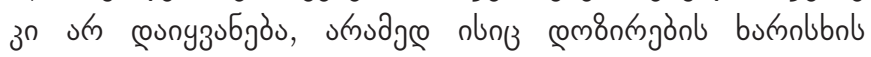

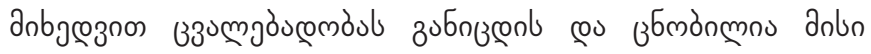

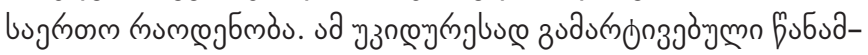

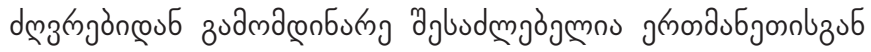

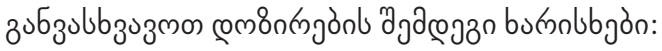

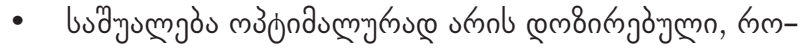

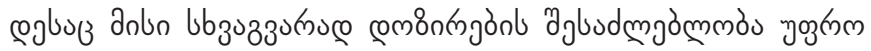

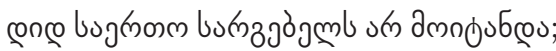

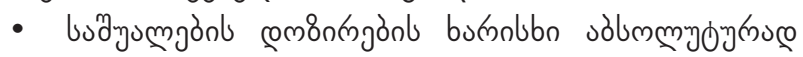

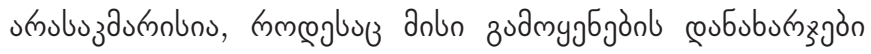

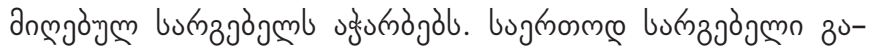

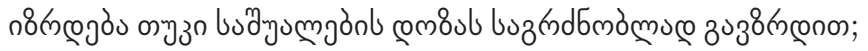

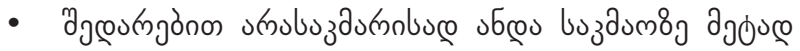

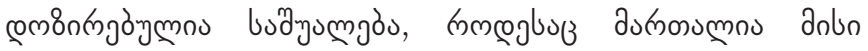

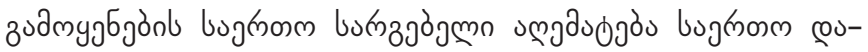

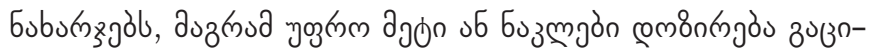

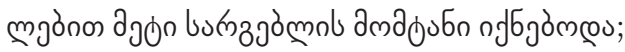

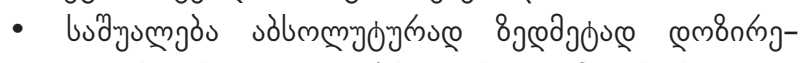

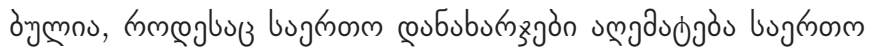

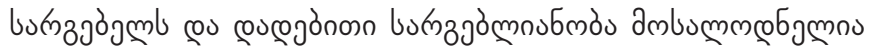

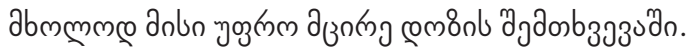

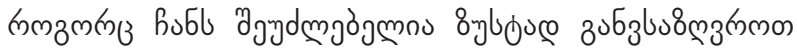

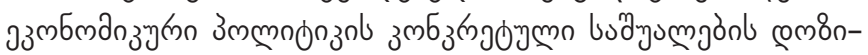

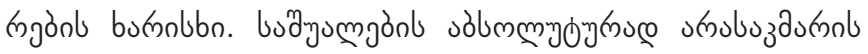

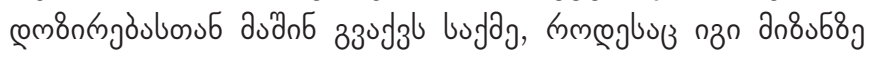

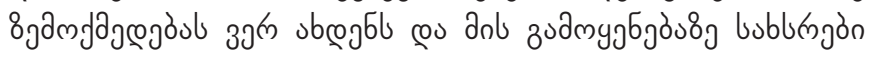

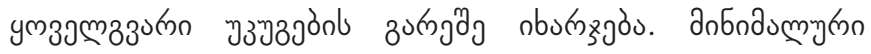

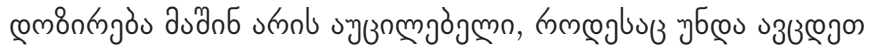

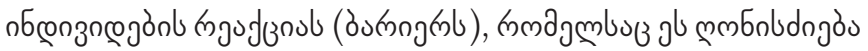

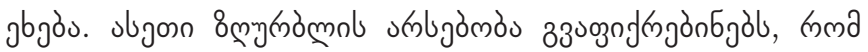

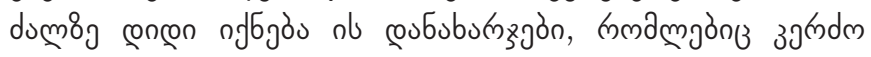

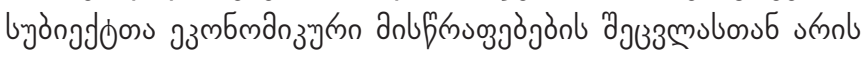

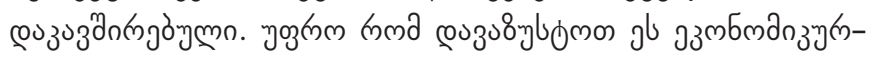

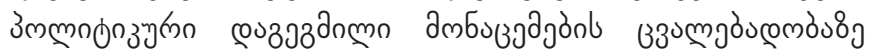

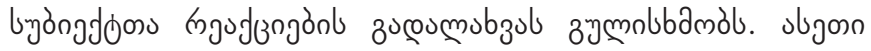

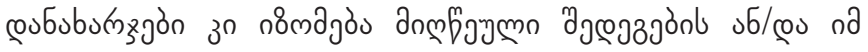

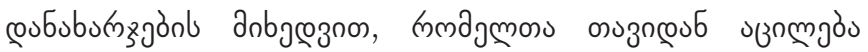

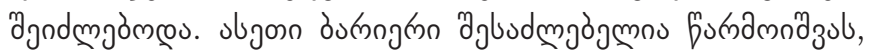

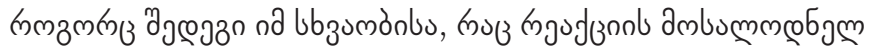

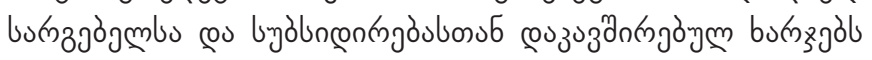

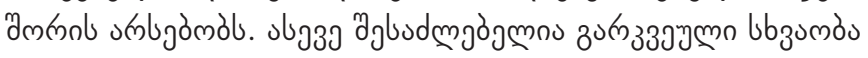

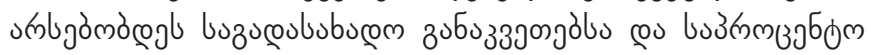

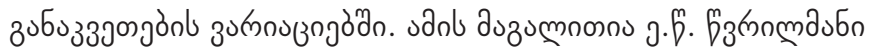

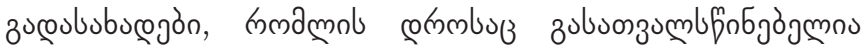

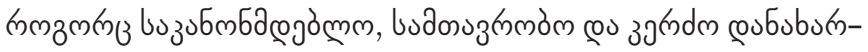

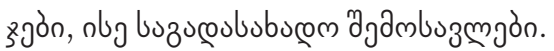




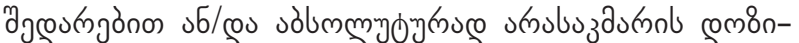

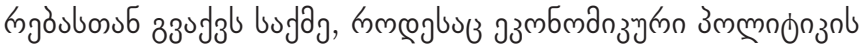

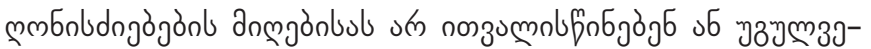

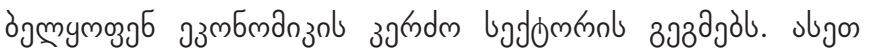

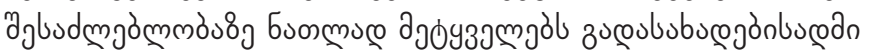

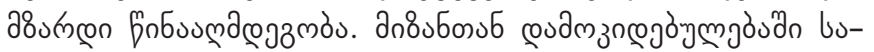

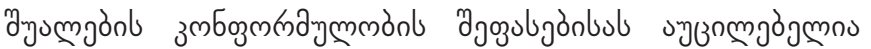

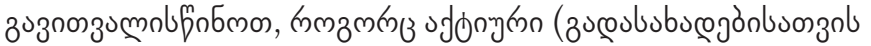

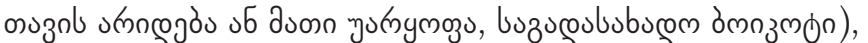

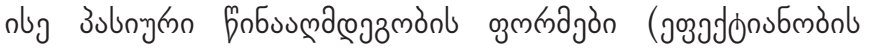

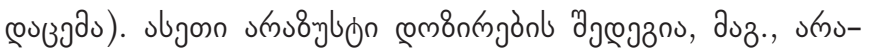

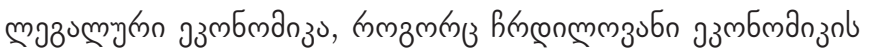
Euponn.

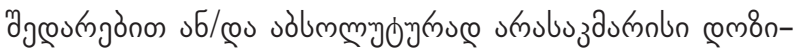

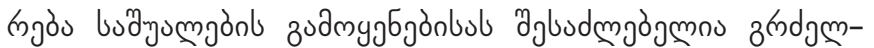

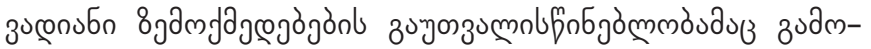

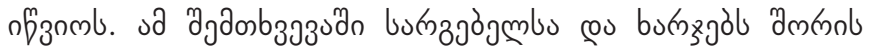

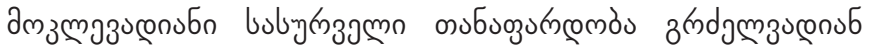

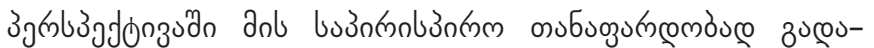

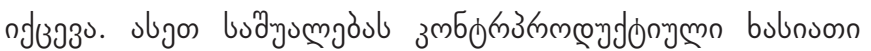

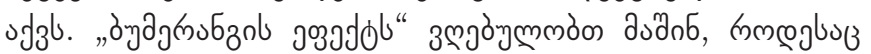

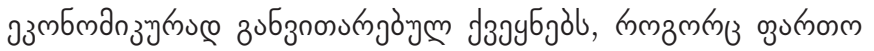

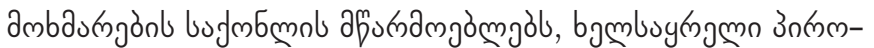

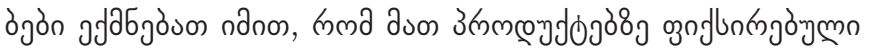

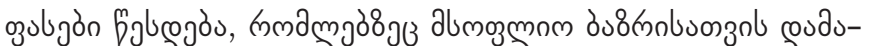

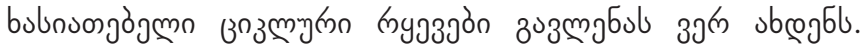

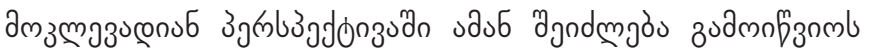

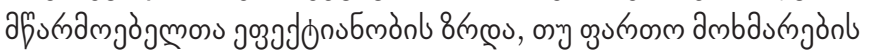

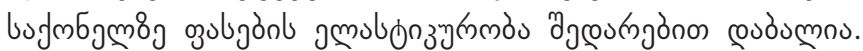

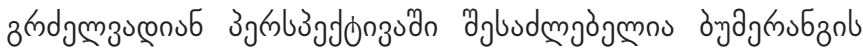

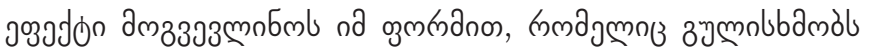

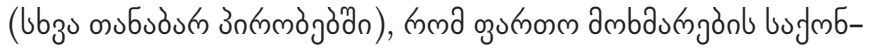

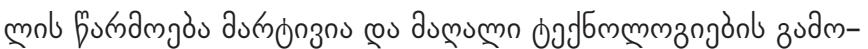

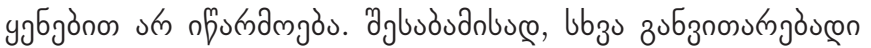

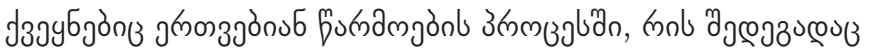

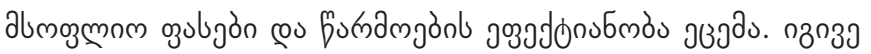

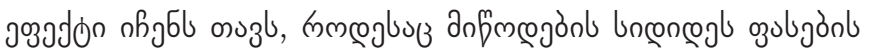

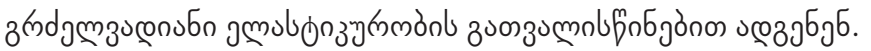

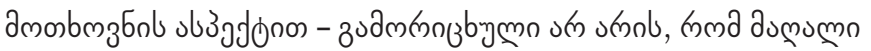

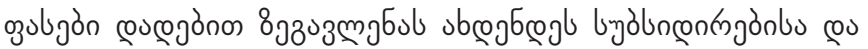

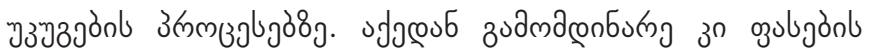

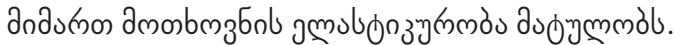

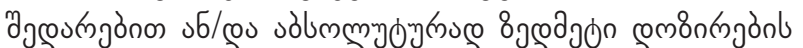

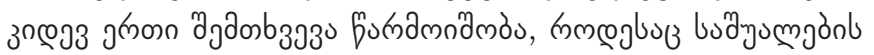

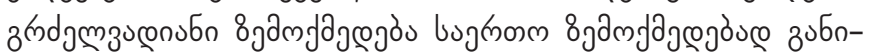

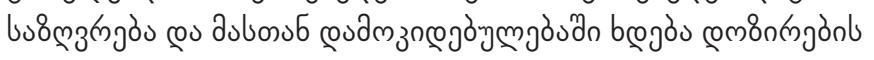

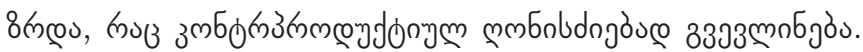

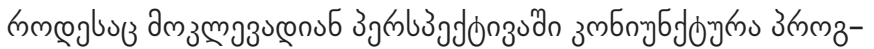

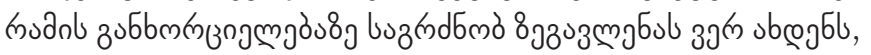

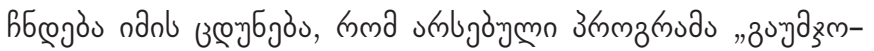

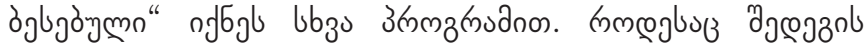

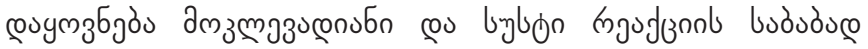

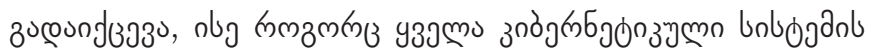

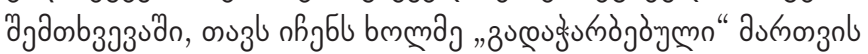

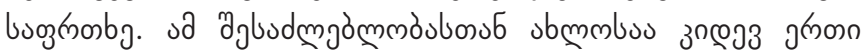

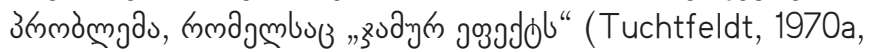

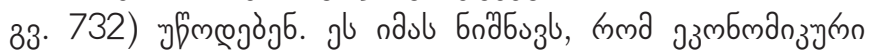

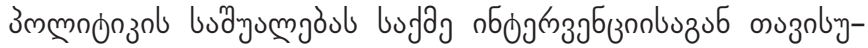

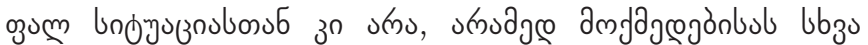

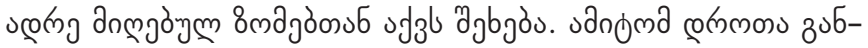

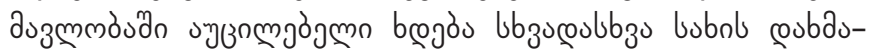

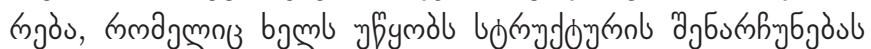

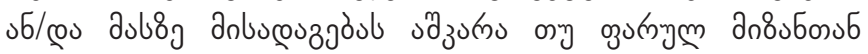

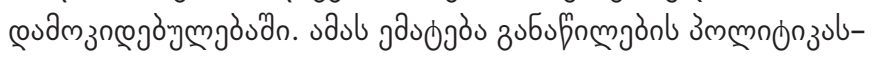

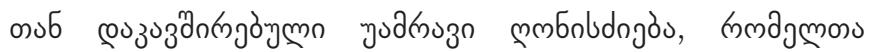

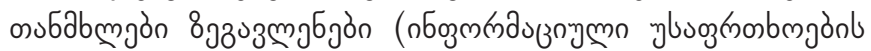

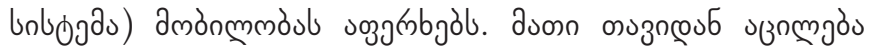

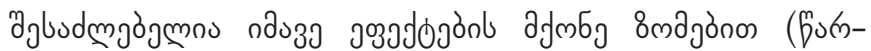

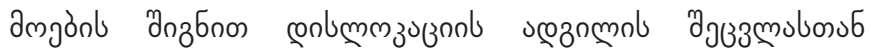
एозз

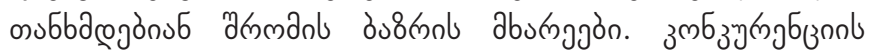

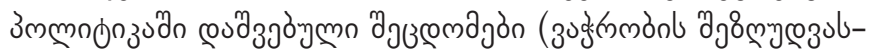

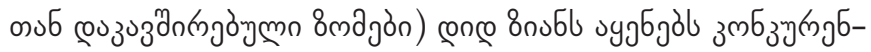

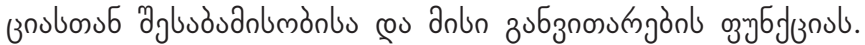

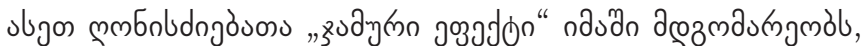

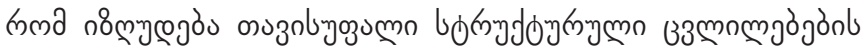

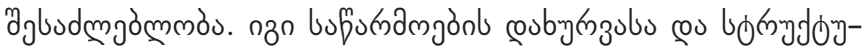

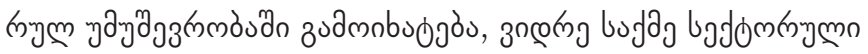

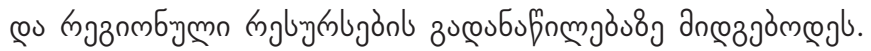

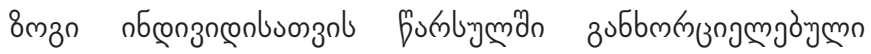

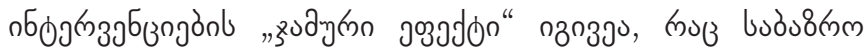

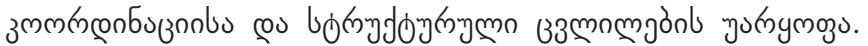

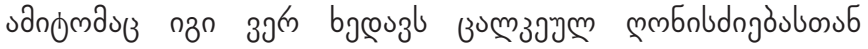

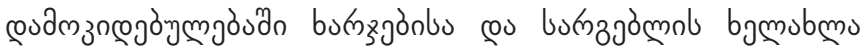

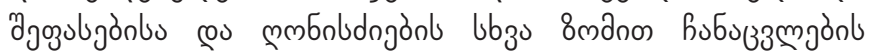

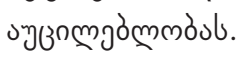

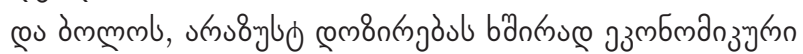

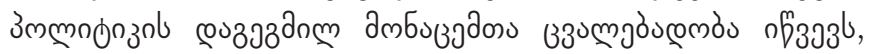

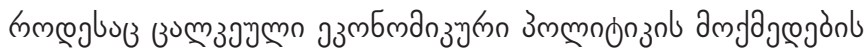

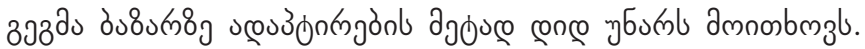

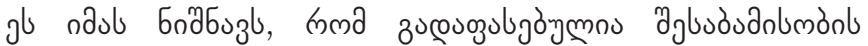

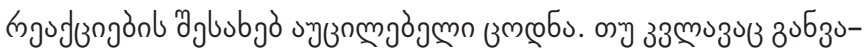

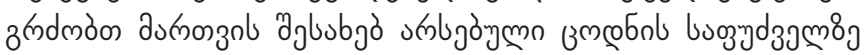

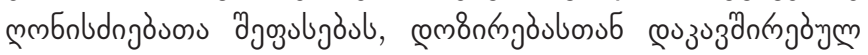
з६омдпмлды

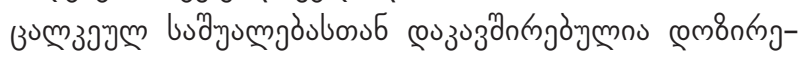

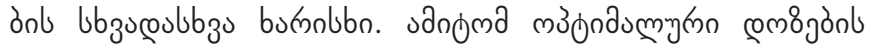

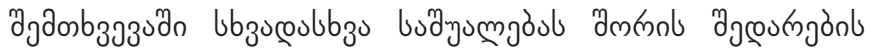

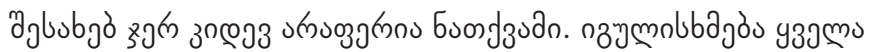

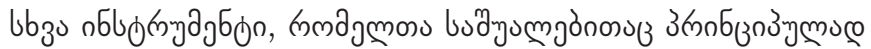




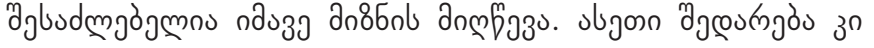

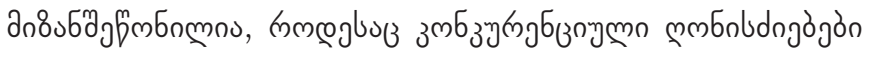

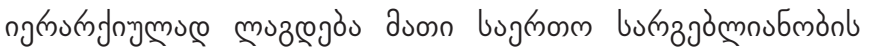

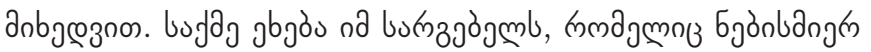

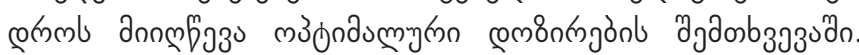

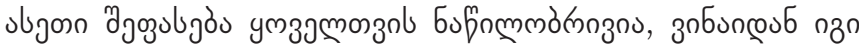

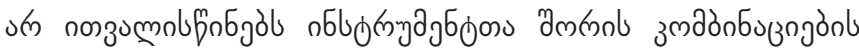

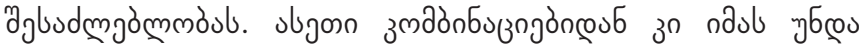

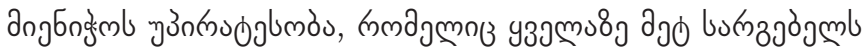

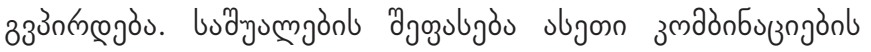

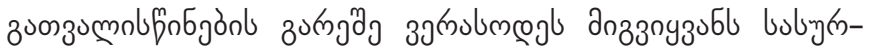

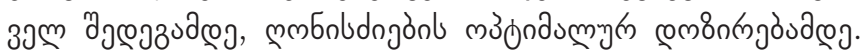

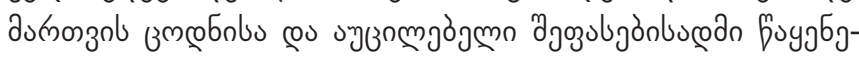

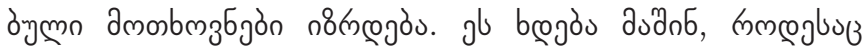

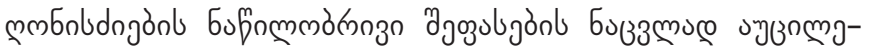

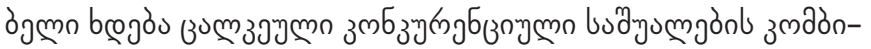

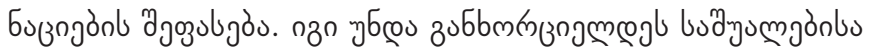

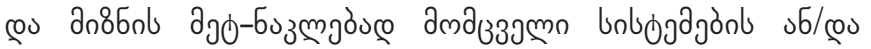

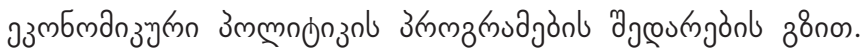

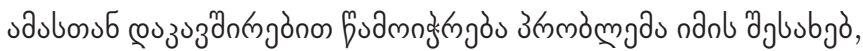

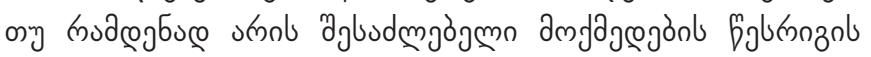

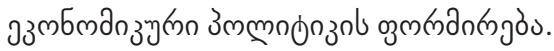

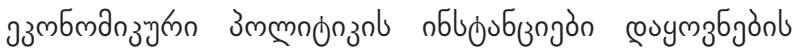

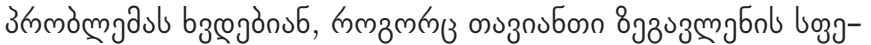

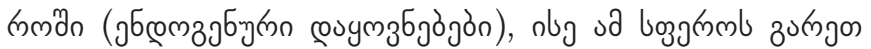
(

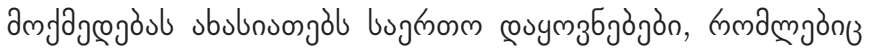

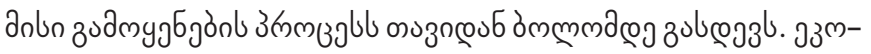

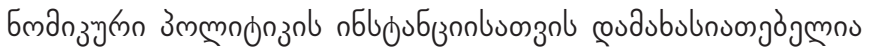

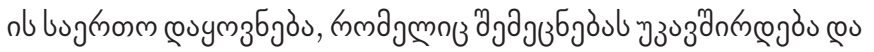

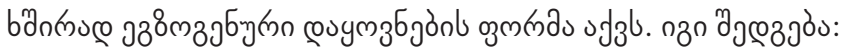

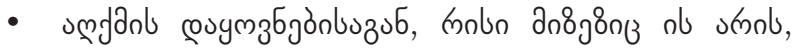

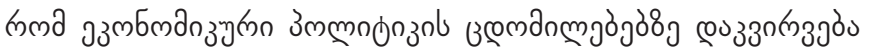

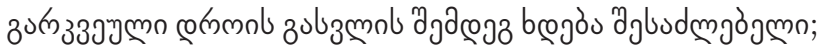

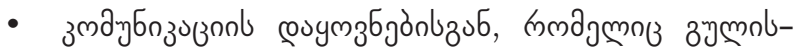

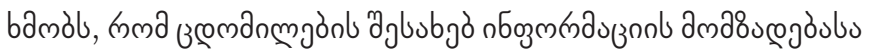

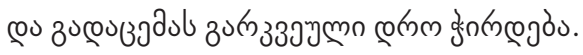

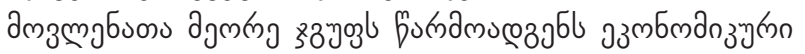

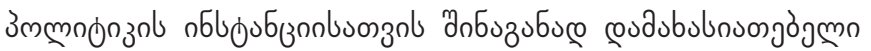
cougm 3 бुojgòn:

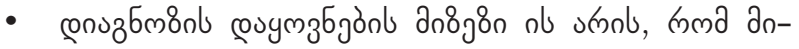

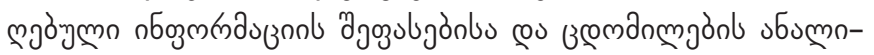

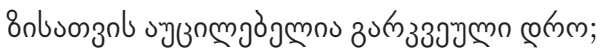

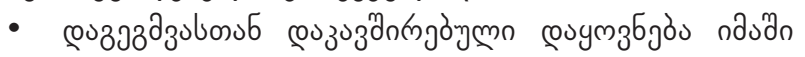

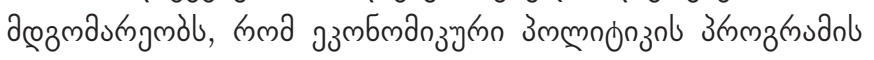

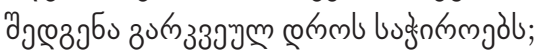

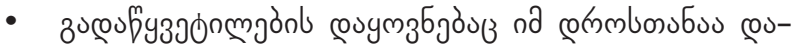
з З

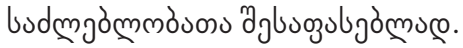

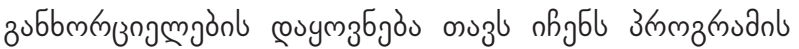

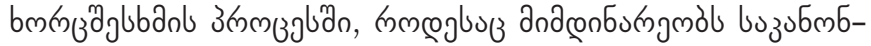

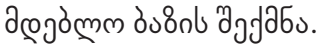

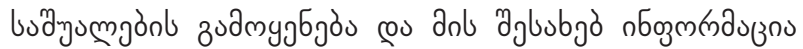

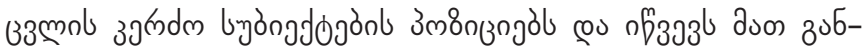

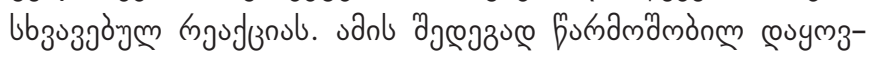

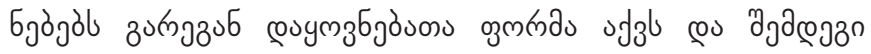

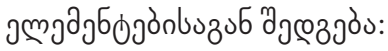

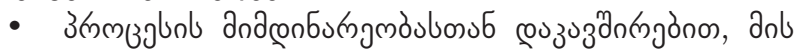

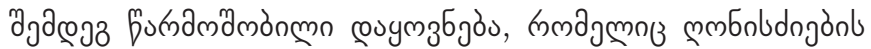

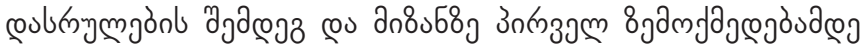
nfjбl bmman os 3 b;

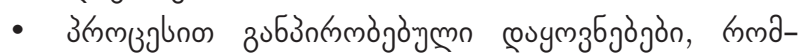

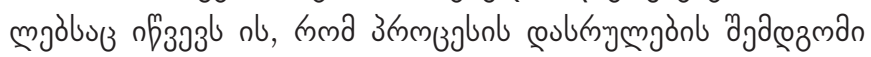

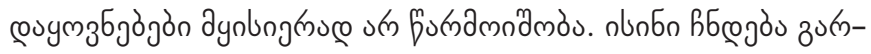

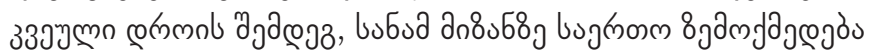
ambejós.

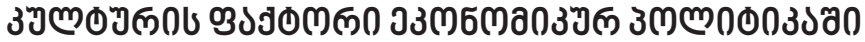

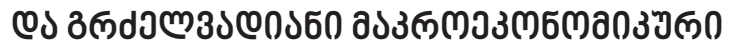

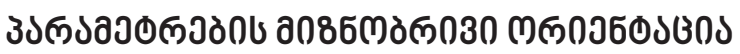

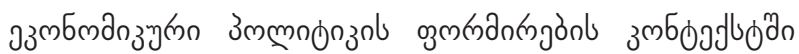

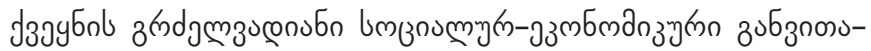

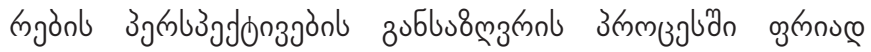

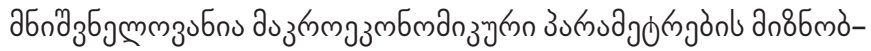

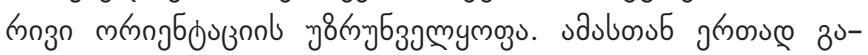

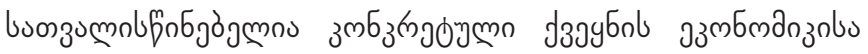

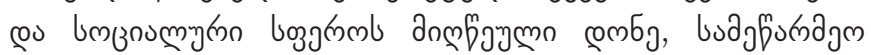

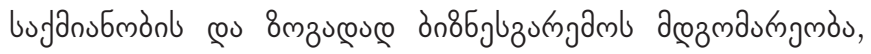

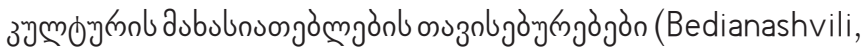
2020).

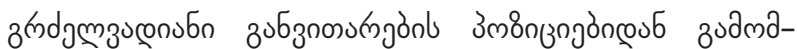

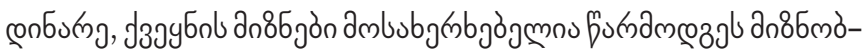

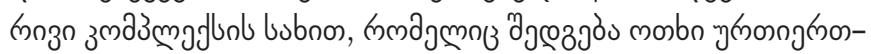

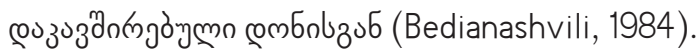

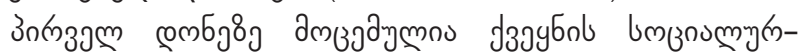
उзмбмдаз з

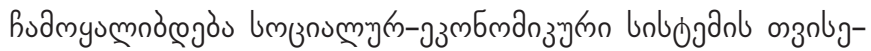

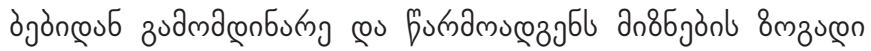
оुмn

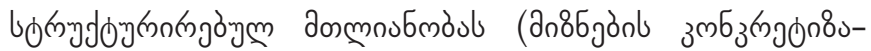

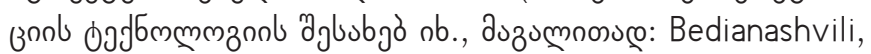

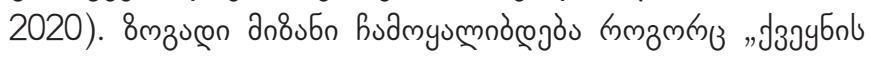

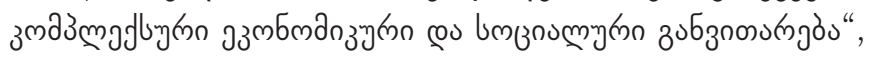

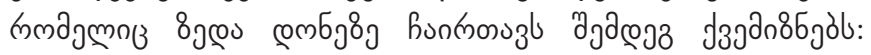

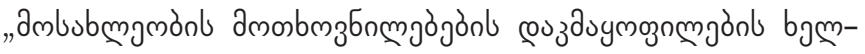

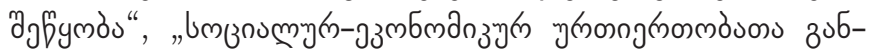

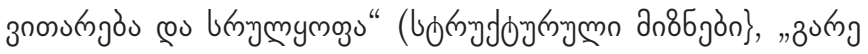

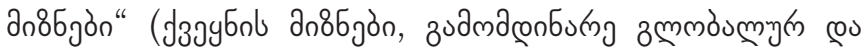




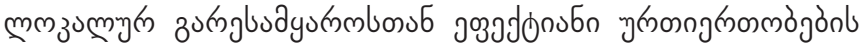

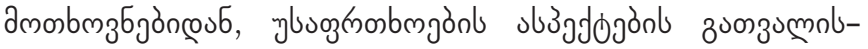

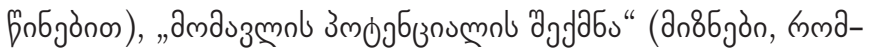

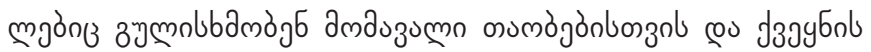

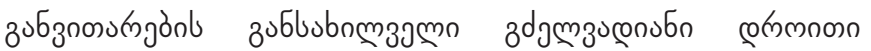

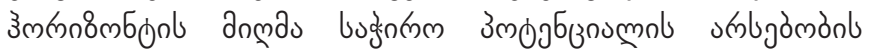

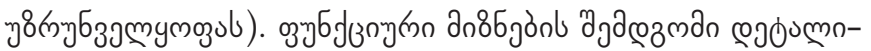

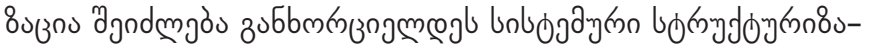

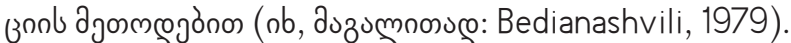

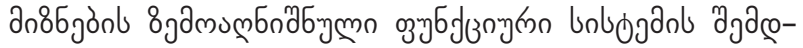

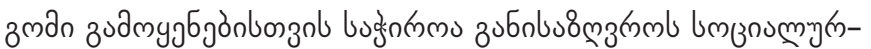

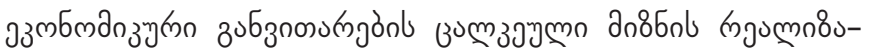

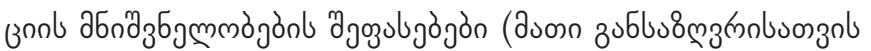

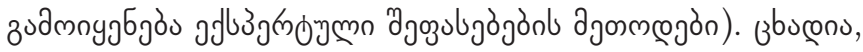

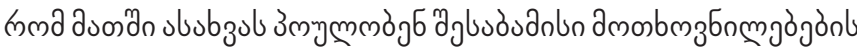

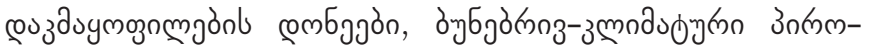

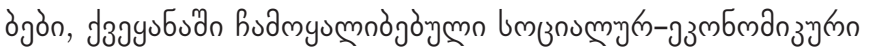

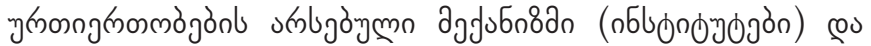

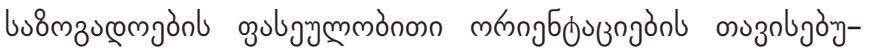

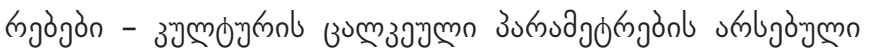

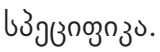

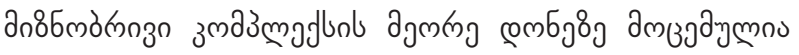

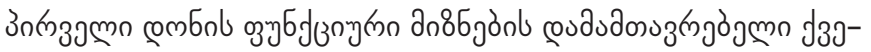

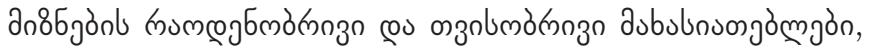

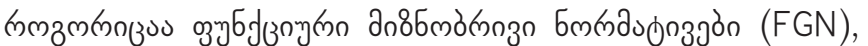

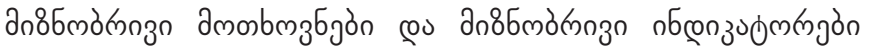

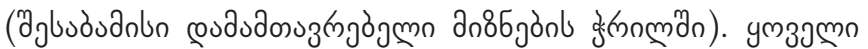

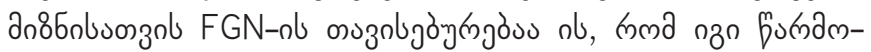
ง

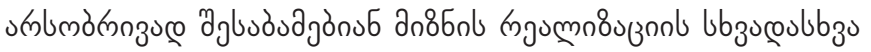

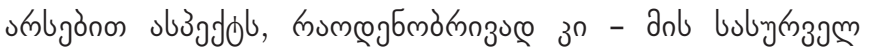

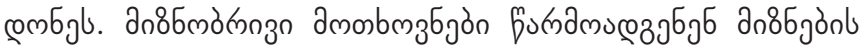

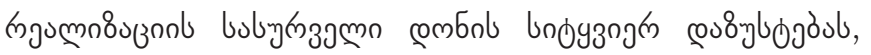

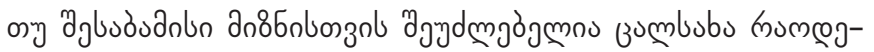

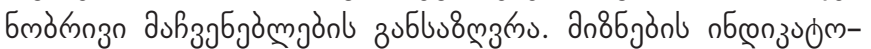

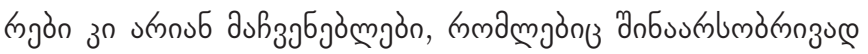

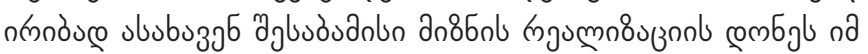
उ ऊmm(z)

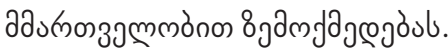

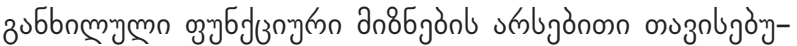

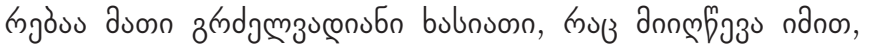

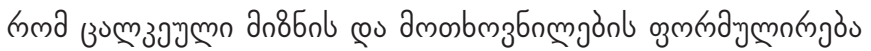

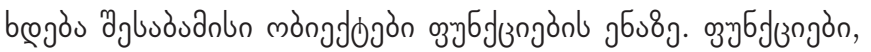

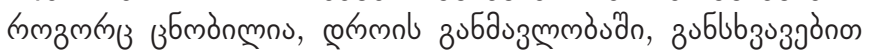

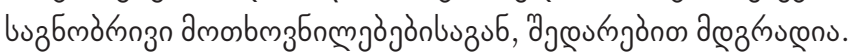

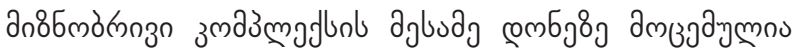

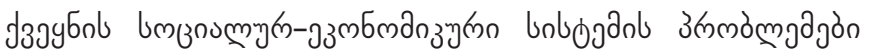

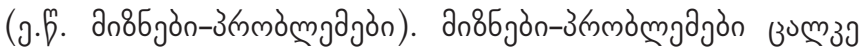

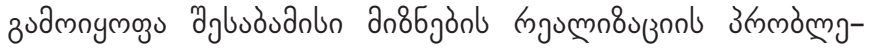

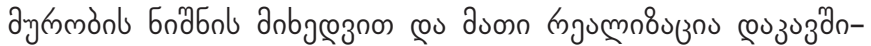

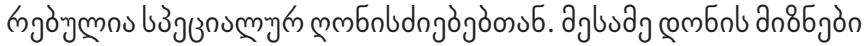

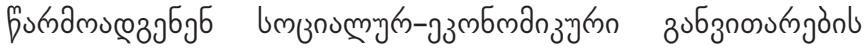

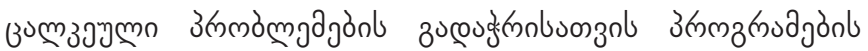

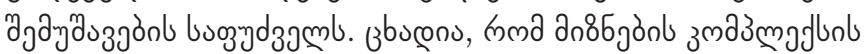

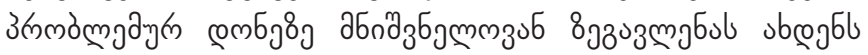

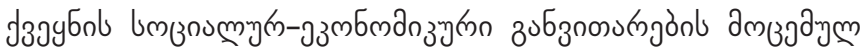

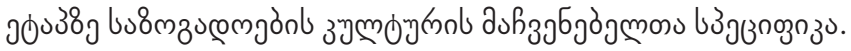

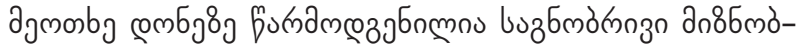

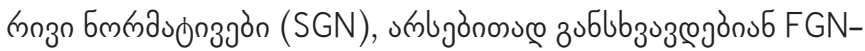

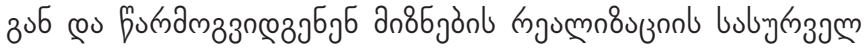

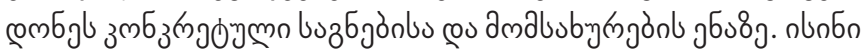

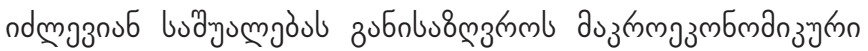

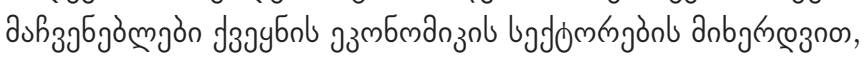

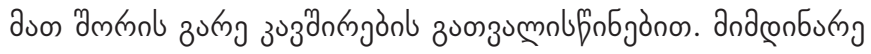

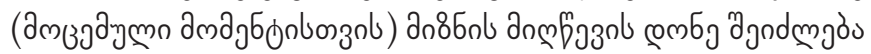

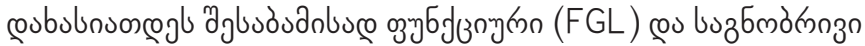

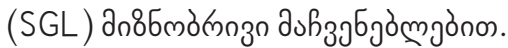

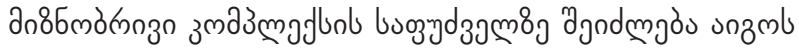

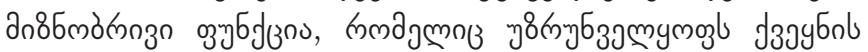

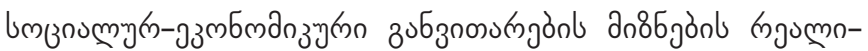

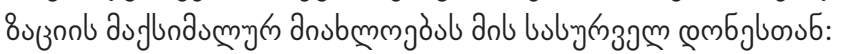

$$
\sum_{i \in I_{f}} \sum_{t \in T} \alpha_{i}\left\|G_{i l}{ }^{(t)}\right\|^{2}+\sum_{i \in I_{n}} \sum_{t \in T} \alpha_{i}\left\|Y_{i l}{ }^{(t)}\right\|^{2} \rightarrow \min
$$

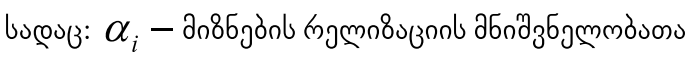

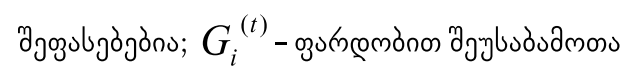

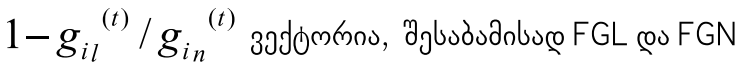

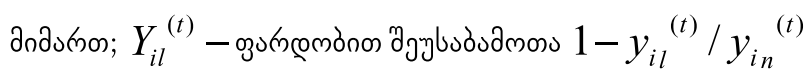

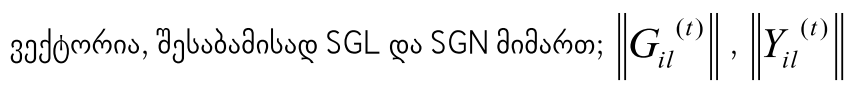

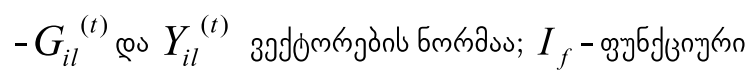

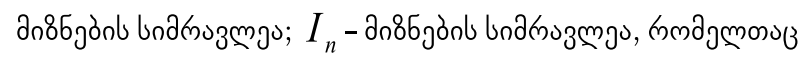
ง

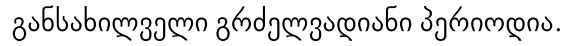

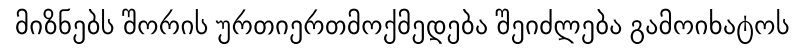

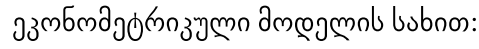

$$
G^{(t)}=\sum_{\sigma=0}^{\bar{\sigma}} A^{(t-\sigma)} G^{(t-\sigma)}+C^{(t)} R^{(t)}+D^{(t)} P^{(t)}+U^{(t)},
$$

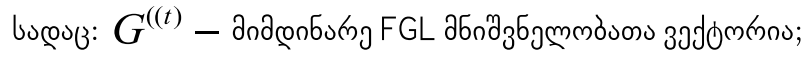

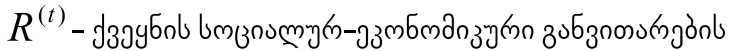

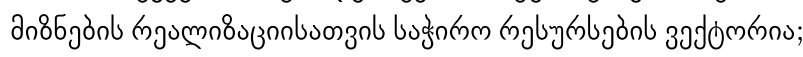

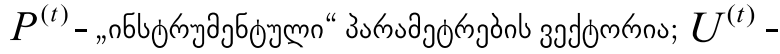

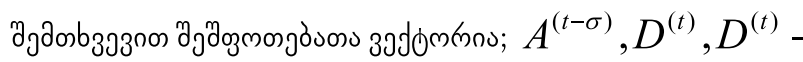
змjoुngn

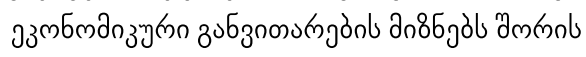

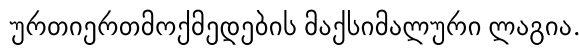




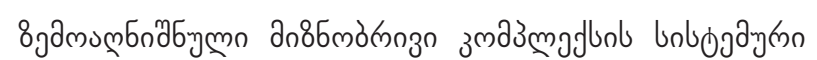

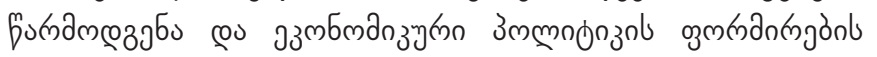

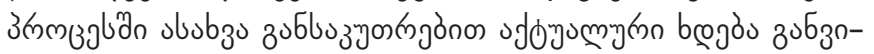

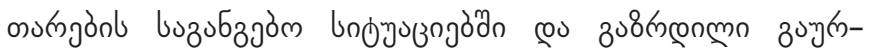

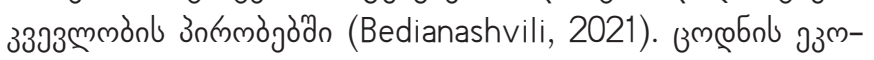

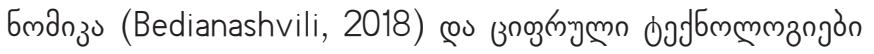

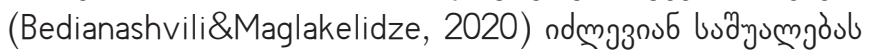

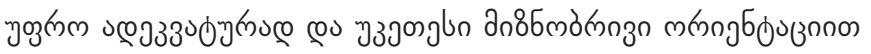

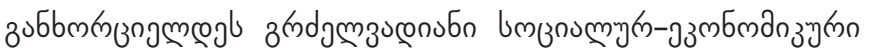

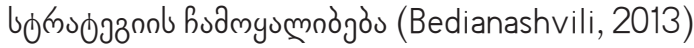

\section{ess336s}

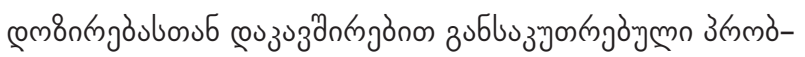

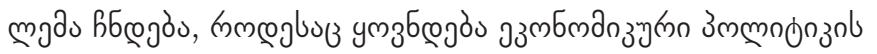
buàyumgonl zudmyjб

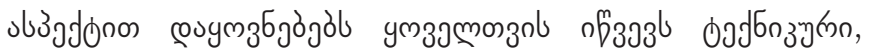

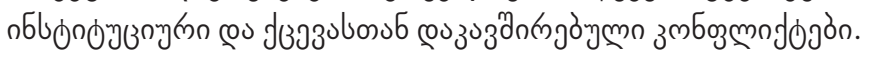

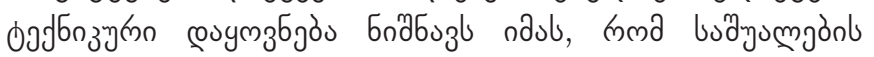

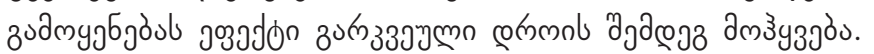

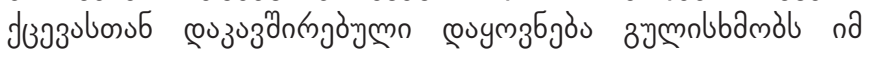

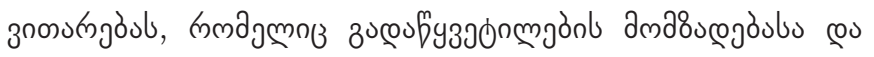

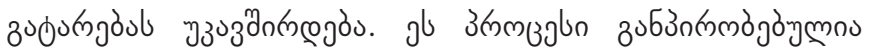

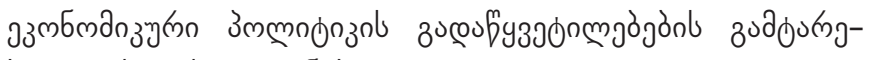

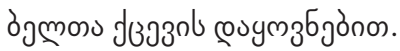

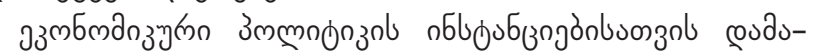

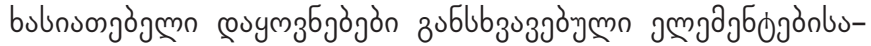

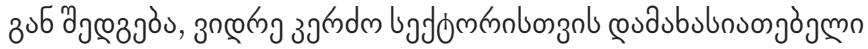

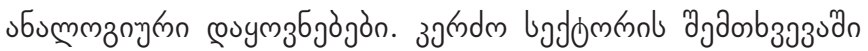

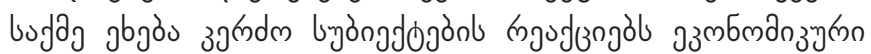

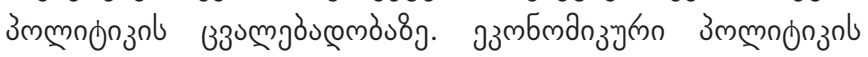

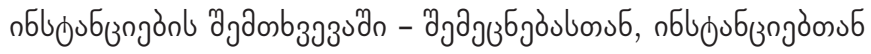

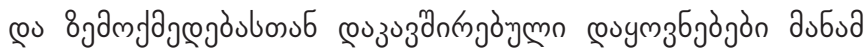

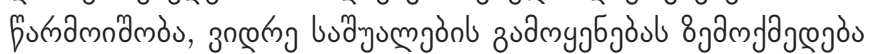

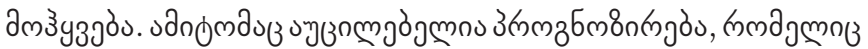

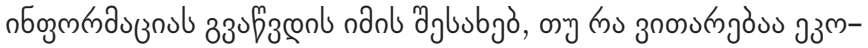

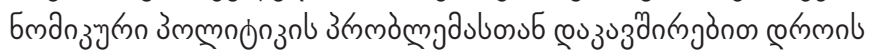

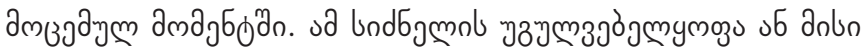

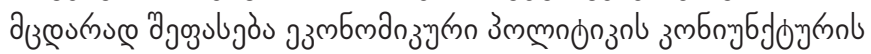

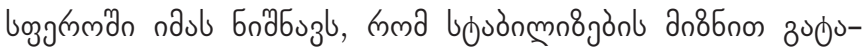

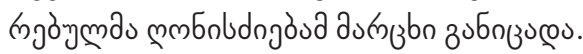

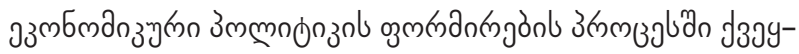

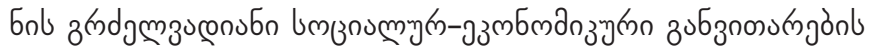

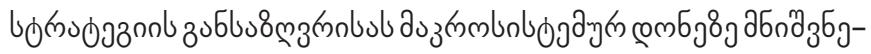

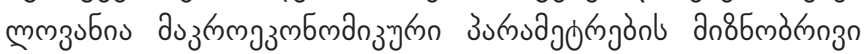

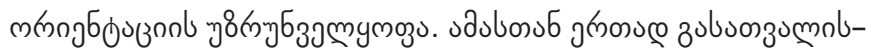

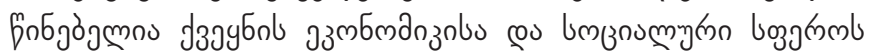

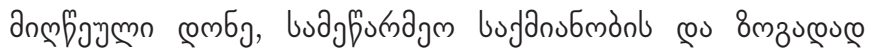

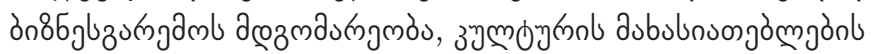

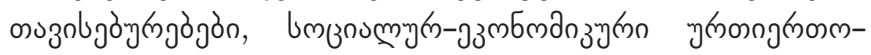
ajôn u v

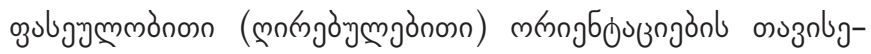

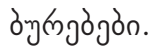

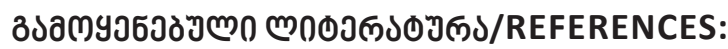

Bedianashvili, G. (2021). Macroeconomic and Cultural Determinants of the COVID-19 Pandemic Crisis. Bulletin of the Georgian National Academy of Sciences, 15 (2): 191-197.

Bedianashvili, G., Maglakelidze, A. (2021). The Digital Economy and Business in the Conditions of Pandemic. Conference Materials: Social Sciences for Regional Development 2020. Daugavpils University. Part III. Issues of Economics: 26-37.

Bedianashvili, G. (1984). The Goal Aspect of the Long-Term Socio-Economic Development of Region. Bulletin of the Georgian National Academy of Sciences. 113 (1): 189-192 (In Russian).

Bedianashvili, G. (2018). Knowledge Economy, Entrepreneurial Activity and Culture Factor in Modern Conditions of Globalization: Challenges for Georgia. Globalization and Business, 5: 32-37.

Bedianashvili, G. (2013). Strategy of Long-Term Socioeconomic Development of the Country: Issues of Methodology. Conference materials: Actual Problems of Economies of Post-Communist Countries at Current Stage. Ivane Javakhishvili Tbilisi State University Paata Gugushvili Institute of Economics, Georgia, Tbilisi.:121-125 (In Georgian).

Boehm, E. (1966), Privatrechtsgesellschaft Und Marktwrstchaft Ordo 17, S.75-151. P. 80

BOULDING, K.E. (1962), Knowledge as a Commodity; Wiederabgedruckt In Ders. (Hrsg.): Beyond Economics - Essays on Society, Religion, And Ethics, Ann Arbor 1968, P.141-150

Dahl, R.A. (1963), Die Politische Analyse (Uebersetzung Von „,Modern Political Analysis,” Englewood Cliffs), Muenchen 1973, P. 62.

Gaprindashvili, G. (2017). "The Role of Government's Economic Policy In Country's Economic Development", Journal Association 1901 "SEPIKE”, Social Educational Project Of Improving Knowledge In Economics. Vol. 18, Pp.121-127.

Gaprindashvili, G. (2019). The Results of State Procurement Reforms In Georgia, 2nd International Conference On Business, Management And Economics. Vienna, Austria, Pp.213-225

Gaprindashvili, G.(2020) STATE PROCUREMENT ASPECTS IN GEORGIA, Ecoforum Journal,Volume 9, Issue 1. 
Gvelesiani R., Gogorishvili I. (2014), The Basic Problems For The Realization Of The Concept Of Economic Policy. XII International Conference On Economics And Management, International Science Index, Vol. 8. No 11; November 10-11, Madrid, Spain, P. 532-535.

Gvelesiani R. (2015a), Intrinstic Contradictions of Entrepreneurship Development And Self-Development, XIII International Conference On Economics, Business And Marketing Management, International Science Index, Eissn: 1307-6892, Vol: 9 No:3, March 26-27, Madrid, Spain, P.1344-1346.

Gvelesiani R. (2015b), The Influence Of Interest Groups On Economic Policy And Its Contradictory Results, Journal Of Academy Of Business And Economics, IABE, Volume 15, № 2, ISSN: 1542-8710, Roma Tre University, Italy, Editor: South Stockholm University, Sweden, P.35-40.

Gvelesiani R. (2016a), Individuals and Economic Group's Interests and Behaviour in the Economic and Political Decision-Making Process, European Journal of Business Research (EJBR), IABE, Volume 16, №1. ISSN: 1945-2977, University of Pisa, Italy, Editor: Johannes Kepler University Linz, Austria, P. 97-103.

Gvelesiani R. (2016b), Strenghtening of the Tendency Towards Social Entrepreneurship as a Demonstration of the Potential for the Improvement of Modern Society's Moral Characteristics, International Journal of Strategic Management (IJSM), IABE, Volume 16, № 2, ISSN: 1555-2411. University Of Florence, Italy, Editor: Trinity Western University, Canada, P. 17-22.

Gvelesiani R. (2017a), Possibilities of Implementing the Practical Economical Politics Rationally, European Journal of Business Research $^{\text {tm }}$ (EJBR), Volume 17, №2, ISSN: 1945-2977, Dx.Doi.Org/10.18374/EJBR-17-2.3, New York Institute Of Technology, USA, Editor-In-Chief: Professor Christine Duller, Johannes Kepler University Linz, Austria, P.25-30.

Gvelesiani R. (2017b), Social Inequality - The Revealing Form of Conflicts Among the Main Societal Values, Journal of International Management Studies (JIMS), Volume 17, \#1, ISSN: 1930-6105, University of Sassari, Italy, Editor: Trinity Western University, Canada, P.59-64.

Gvelesiani R. (2017c), The Problem of Considering Possible Consequences of The Consistent Reforms in The Economic-Political Decisions, Review of Business Research (RBR), Volume 17, №2, , ISSN: 1546-2609, University Of Sassari, Italy, Editor: University Of Osijek, Croatia, P.19-24.

Gvelesiani R., (2020a). Contradictory Interdependence Between The Goals Of Economic Policy And Assessment of Its Expected Results, ISSN: 2344-2174, Ecoforum, Vol. 9, No 3.

Gvelesiani R., (2020b). The Problem of Compatibility of Economic Policy Objectives, Instruments and Order Policy Principles, ISSN: 2344-2174, Ecoforum, Vol. 9, No 3.

Gvelesiani, R. (2020c). Contradictions of Public Values - Origin of Conflict of Interests, ISSN: 2344-2174, Ecoforum, Vol. 9, No 2, 2020, Stefan Cel Mare University Of Suceava, Romania. File:///C:/Users/User/Downloads/1084-3445-1-PB.Pdf

Gvelesiani, R. (2020d). Formation of the Innovative Entrepreneurship Culture: Capabilities and Problems, ISSN: 2344-2174, Ecoforum, Vol. 9, No 1, 2020, Sttefan Cel Mare University of Suceava, Romania. http://www.Ecoforumjournal.Ro/Index. $\mathrm{Php} /$ Eco/Index

Gvelesiani, R. (2021). Problems of the Optimal Use of Economic Policy Instruments. Ecoforum, Vol. 10, No 2. http://www.ecoforumjournal.ro/index.php/eco/article/view/1250/756

Hayek, F.A. (1960), The Constitution of Liberty, Neudruck, London U.A. Wiederabdruck In: BOSCH, A. Und R. VEIT Hrsg.), Hayek Gesammelte Schriften Bd. B3, Die Verfassung Der Freiheit, Tuebingen 2005, P.134.

Hayek, F.A. (1979), Law, Legislation And Liberty, Volume III - The Political Order Of A Free People, Chicago, London, Wiederabdruck In: VANBERG, V. (Hrsg.), Hayek Gesammelte Schriften Bd. B4, Recht, Gesetzgebung Und Freiheit, Tuebingen 2003. P. 128.

Hayek, F.A. (2003), Rechtsordnung Und Handelnsordnung, In: STREIT, M. E. (Hrsg.), Hayek Gesammelte Schriften Bd. A4, Rechtsordnung Und Handelnsordnung, Tuebingen. P. 217.

Hayek, F.A. (2004), Der Weg Zur Knechtschaft, In: STREIT, M. E. (Hrsg.), Hayek Gesammelte Schriften Bd. B1, Tuebingen.

Jonas, H. (1979), Das Prinzip Verantwortung, Nachdruck, Frankfurt A. M, 2003. P.294; 393.

Kirsch, G. (1977), Technischer Fortschrift III - Foerderung Durch Die Oeffentliche Hand, In: Handwoerterbuch Der Wirtschaftswissenschaft, Bd. 7, Stuttgart, New York, S.609-620.

Mestmaecker, E.-J. (1975), Wirtschaftsordnung Und Staatsverfassung; In Ders. Und H. SAUERMANN (Hrsg.): Wirtschaftsordnung Und Staatsverfassung (Festschrift Fuer Franz Boehm Zum 80. Geburtstag), Tuebingen, P.383-419).

Mill, J.S. (1859), On Liberty; In Ders.: Utilitarism, Liberty, Representative Government, Neuausgabe, Hrsg. V. H. B. ACTON, London 1972, P. 75.

Samuelson, P.A. Und Solow R., M. (1960), Analytical Aspects Of Anti-Inflation Policy. American Economic Review 50, Papers and Proceedings, P. 177-194.

Streit, M. E. (2005), Theorie Der Wirtschaftspolitik, 6. Auflage, Stuttgart, S. 307; 309; 310.

Tuchtfeldt, E. (1970a), Ueber Unerwuenschte Nebenwirkungen Wirtschaftspolitischer Mssnahmen. Kyklos 23, S. $720-735$.

Tuchtfeldt, E. (1982), Wisrtchaftspolitik, In: Handwoerterbuch Der Wirtschaftswissenschaft, Bd. 9, Stuttgart U. A., S. $178-206$.

Weber, M. (1921), Wirstchaft Und Gesellschaft, 5. Rev. Aufl., Studienausg., Nachdruck, Tuebingen, 2002. P. 28 